\title{
Le nouveau paradigme de la violence (Partie 1)
}

\author{
Michel Wieviorka
}

\section{OpenEdition}

\section{Journals}

\section{Édition électronique}

URL : http://journals.openedition.org/conflits/724

DOI : $10.4000 /$ conflits.724

ISSN : 1777-5345

Éditeur :

CCLS - Centre d'études sur les conflits lilberté et sécurité, L'Harmattan

\section{Édition imprimée}

Date de publication : 15 octobre 1998

ISSN : 1157-996X

Référence électronique

Michel Wieviorka, «Le nouveau paradigme de la violence (Partie 1)», Cultures \& Conflits [En ligne], 29-30 | automne-hiver 1998, mis en ligne le 16 mars 2006, consulté le 30 mars 2021. URL : http:// journals.openedition.org/conflits/724 ; DOI : https://doi.org/10.4000/conflits.724

Ce document a été généré automatiquement le 30 mars 2021.

Creative Commons License 


\title{
Le nouveau paradigme de la violence (Partie 1)
}

\author{
Michel Wieviorka
}

1 La violence n'est pas la même d'une période à une autre. En ce sens, l'historien Charles Tilly apporte un éclairage utile lorsqu'il propose de caractériser chaque grande époque historique qu'il étudie par son " répertoire " spécifique des formes de l'action, et plus particulièrement de la violence ${ }^{1}$. Or précisément, les transformations récentes, depuis les années soixante et soixante-dix, sont si considérables qu'elles justifient d'explorer l'idée de l'entrée dans une ère nouvelle, et, de là, celle d'un paradigme nouveau de la violence, qui caractériserait le monde contemporain. Qu'il s'agisse des manifestations tangibles du phénomène, des représentations qui en circulent, ou de la façon dont les sciences sociales l'abordent, de si profonds changements sont en effet en jeu qu'il est légitime de mettre l'accent sur les inflexions et les ruptures de la violence, plus que sur des continuités qu'il n'est pas question pour autant de sous-estimer. Ajoutons qu'en laissant de côté, comme nous le ferons ici, la question du perfectionnement technologique et scientifique dans le domaine des armes, nous nous privons d'éléments qui vont certainement dans le sens de l'idée d'un nouveau paradigme ${ }^{2}$.

De nouvelles significations

4 La violence, aujourd'hui, s'est fortement renouvelée dans les significations de ses expressions les plus concrètes, et nous insisterons ici, pour l'essentiel, sur les changements qui l'informent depuis la fin des années soixante. Une perspective inscrite dans la plus longue durée, prenant par exemple pour période de référence le siècle écoulé, ne mettrait vraisemblablement pas en défaut l'hypothèse d'un nouveau paradigme que nous allons examiner; elle suggérerait simplement, peut-être, que parmi les significations les plus décisives d'aujourd'hui, quelques-unes ressemblent à celles qui ont caractérisé l'entrée dans l'ère industrielle, lorsque les classes contestataires naissantes étaient surtout perçues comme des classes dangereuses ou que, dans un pays comme la France, des phénomènes de bandes et des conduites de violence juvénile imputées aux " Apaches " défrayaient la chronique ${ }^{3}$ et étaient traités 
par la presse en des termes qui font penser à ce qui se dit aujourd'hui des jeunes en colère dans les quartiers de relégation.

Importants dans les années soixante-dix et encore quatre-vingt, la violence politique et le terrorisme d'extrême-gauche, liés à la longue déstructuration des idéologies, des régimes et des partis d'inspiration marxiste-léniniste, ainsi qu'à un refus de plus en plus artificiel de percevoir le déclin historique du mouvement ouvrier, ont partout régressé ; Action Directe, Brigades Rouges, Cellules Révolutionnaires, Fraction Armée Rouge, etc., ce type d'organisation est à peu près partout dans le monde épuisé, presque liquidé historiquement - ce qui ne veut pas dire qu'il faut exclure, à l'avenir, le retour d'idéologies marxistes-léninistes et de violences s'en inspirant, comme on le voit déjà aujourd'hui au Mexique, où la guérilla de l'EPR (Exercito Popular Revolucionario) s'inscrit dans des orientations qui font à bien des égards penser aux années soixante ou soixante-dix. Presque symétriquement, la violence d'extrême-droite, animée par des projets de prise du pouvoir d'Etat, a elle aussi régressé, souvent remplacée par des conduites qui visent non plus à assurer à leurs acteurs le contrôle de l'Etat, mais à l'inverse, à tenir l'Etat à distance de pratiques privées. L'expérience italienne est ici un cas spectaculaire. Dans les années soixante-dix et jusqu'au milieu des années quatrevingt, les terrorismes d'extrême-gauche et d'extrême-droite voulaient dans ce pays, l'un en finir avec l'" Etat impérialiste des multinationales ", l'autre créer le climat favorable à un coup d'Etat à l'aide d'une " stratégie de la tension ". Depuis, les violences majeures contre l'Etat ont été avant tout destinées à protéger les activités économiques privées de groupes mafieux.

6 A partir des années cinquante, des luttes de libération nationale, éventuellement associées à des orientations marxistes-léninistes et pouvant prendre l'allure d'une guérilla ont souvent donné naissance à de nouveaux régimes et de nouveaux Etats ; leur violence n'est plus aujourd'hui aussi importante, à l'échelle de la planète, que dans les années cinquante à soixante-dix, même si certaines d'entre elles se perpétuent, par exemple en Europe avec les expériences basque ou d'Irlande du nord, ou bien au Moyen Orient, avec le mouvement palestinien, et si d'autres se sont affirmées récemment, notamment en Tchétchénie. Ce constat ne veut pas dire qu'on assiste à la dissolution des liens associant éventuellement violence et nation, car ceux-ci peuvent revêtir d'autres significations que celles qu'implique le thème de la libération nationale. En effet, le nationalisme, y compris au coeur des pays les plus puissants, constitue un phénomène contemporain majeur, souvent lié à une thématique d'extrême-droite, mais moins directement lié à des expressions caractérisées de violence que ce que suggère le discours spontané : en Europe tout au moins, le national-populisme et la montée en force des droites radicales sont des phénomènes qui dans l'ensemble ne s'accompagnent guère de violence, tout simplement parce que celle-ci, même si elle peut surgir ça et là, est vite contradictoire avec la respectabilité qu'appelle un projet d'accès au pouvoir par la voie électorale. La violence nationaliste, de ce point de vue, est limitée, souvent plus ethnique, voire raciale, que proprement nationaliste, et associée non pas tant à l'idée d'assurer la libération d'une nation, qu'au souci de la protéger de menaces extérieures et de la purger de tout ce qui entacherait son homogénéité. Hier, force considérable d'émancipation, l'idée de nation s'associe aujourd'hui non pas tant à des conduites violentes qu'à des idéologies réactives portées par des communautés ou par certains de leurs segments soucieux de fermeture économique et de pureté culturelle, voire raciale ${ }^{4}$. 
7 Le déclin du mouvement ouvrier et la perte de centralité des rapports de production industriels rendent improbables l'idée d'un lien entre d'importantes violences sociales et l'insertion de leurs acteurs dans un conflit structurel de classe, au sens habituel de l'expression. Ce n'est plus la lutte contre l'exploitation, le soulèvement contre un adversaire qui entretient avec les acteurs une relation de domination, mais plutôt le non-rapport social, l'absence de relation conflictuelle, l'exclusion sociale, éventuellement lestée de mépris culturel ou racial, qui alimentent aujourd'hui partout dans le monde, y compris en Europe occidentale, des conduites émeutières ou une violence sociale plus diffuse, portée par la rage et les frustrations. Dans ce contexte, la violence n'est pas seulement un ensemble de pratiques objectives; elle est aussi une représentation, un attribut que par exemple des groupes, parmi les plus aisés, affectent éventuellement, et de manière plus ou moins fantasmatique, à d'autres groupes, généralement parmi les plus démunis.

Enfin, l'élément le plus spectaculaire du renouveau de la violence aujourd'hui est donné par la montée en puissance des références de ses protagonistes à une identité ethnique ou religieuse. Celles-ci constituent une ressource culturelle éventuellement mobilisée de manière violente à des fins politiques, elles alimentent parfois aussi une barbarie homicide allant aux extrêmes, bien au-delà d'enjeux simplement politiques. De telles références, lorsqu'elles sont effectivement portées par des acteurs, et non pas imputées abusivement par les médias ou l'opinion à des conduites qui n'ont en réalité rien à voir avec elles, peuvent avoir l'allure de résurgences, comme si l'espace de violences traditionnelles, ou classiques, ne faisait que s'élargir sous l'effet de conditions favorables. En fait, malgré une allure effectivement traditionnelle, voire fondamentaliste, ce sont des constructions historiques souvent récentes, qui n'ont rien de naturel, et que Jean-François Bayart analyse bien lorsqu'il explique qu'elles relèvent pour l'essentiel de la stratégie rationnelle d'une part, de rêves et de cauchemars d'autre part, " auxquels nous adhérons parce qu'ils nous enchantent ou nous terrorisent " 5 .

$9 \quad$ Les identités constituent pour l'essentiel une nouveauté, elles sont produites bien plus que reproduites, invention plus que tradition, et Jean Baudrillard a raison d'affirmer qu' "au lieu de déplorer la résurgence d'une violence atavique, il faut voir que c'est notre modernité elle-même, notre hypermodernité, qui produit ce type de violence et ces effets spéciaux dont le terrorisme fait partie lui aussi "6.

Perceptions et représentations

11 La violence change aussi si l'on considère non plus le phénomène dans ce qu'il présente de plus concret, de plus objectif, mais dans les perceptions qui en circulent, dans les représentations qui la décrivent. Au sein des pays occidentaux, à commencer par la France, la violence subjective, telle qu'elle est ressentie, présente pour première caractéristique fondamentale, de sembler aujourd'hui avoir perdu toute légitimité dans l'espace politique, ou presque, au point de signifier le mal absolu; elle est ce que la société, unanime, doit absolument proscrire et combattre, en son sein comme en dehors. Dans les années soixante et soixante-dix, la violence pouvait encore être justifiée ou comprise par des intellectuels, eux-mêmes éventuellement inscrits dans une lignée révolutionnaire, anarchiste ou encore marxiste-léniniste, elle pouvait être théorisée ou soutenue avec une certaine adhésion, et être tolérée dans la sphère politique. Les uns admiraient les guérillas, et faisaient du " Che " leur héros, d'autres exaltaient plutôt la violence sociale, ou s'efforçaient de la susciter et de l'animer. La pensée de Frantz Fanon, centrée sur l'expérience coloniale apportait à l'idée de rupture 
violente une théorisation que Jean-Paul Sartre radicalisait dans sa préface célèbre au livre Les damnés de la terre ${ }^{7}$ - le même Sartre qui, quelques années plus tard, encourageait sur les chemins d'une action violente les " maoïstes " avec qui il débattait ${ }^{8}$. Quelques-unes des réactions au moment de la révolution iranienne, saluée par exemple en France par Michel Foucault, constituent peut-être une ultime expression de ces courants d'opinion et de ces sympathies politiques et intellectuelles à l'égard de processus et d'acteurs ayant recours à la violence et bénéficiant d'une légitimité d'autant plus grande qu'il s'agissait d'apporter une réponse, au demeurant le plus souvent limitée, aux atrocités et aux abus commis par un pouvoir dictatorial ou autoritaire, du type, par exemple, de ceux qui caractérisèrent l'Amérique latine jusque dans les années quatre-vingt. Depuis lors, la zone intellectuelle et politique où la violence pourrait faire l'objet de prises de position compréhensives, voire ouvertes, s'est singulièrement rétrécie, le phénomène est nécessairement la marque de ce qu'il faut refuser, et le consensus est très large. Il n'y a aucun grand débat philosophique, moral, ou éthique au sujet de la violence, et si des voix se font entendre depuis la " société civile " pour demander à l'Etat de faire usage à l'étranger de sa force, par exemple dans des situations dramatiques du point de vue des droits de l'homme, c'est dans une perspective humanitaire, éventuellement combinée à la cause écologiste, en des termes où toute référence positive à la violence est bannie. Les intellectuels, dans un monde qui n'est plus structuré à partir de la bipolarité Est-Ouest, dans des sociétés où le principe de division et de conflit qu'apportaient les rapports de production industrielle est devenu secondaire, confrontés à des identités nationales ou religieuses dont ils répugnent à soutenir les combats, ont dans l'ensemble pris leurs distances par rapport à toute idée de violence. Ce phénomène vaut peut-être moins pour les significations infrapolitiques de la violence, lorsqu'elle est portée par exemple par des jeunes en colère, que pour ses dimensions proprement politiques, il est peut-être moins tranché lorsque la violence peut être associée aux carences ou à la crise du système, et plus net en ce qui concerne la violence visant l'Etat et le pouvoir comme tels.

Ces changements ont partout une implication facile à observer : faute de débat, faute d'acteurs politiques ou intellectuels capables de rompre le consensus relatif à la violence, celle-ci est nécessairement l'objet de perceptions et de représentations qui fonctionnent par excès et par défaut.

13 Par excès: l'altérité, la différence culturelle, religieuse ou autre sont l'objet de fantasmes et de peurs, les acteurs qui sont supposés les incarner sont susceptibles d'être diabolisés, au point qu'il leur est couramment imputé une violence virtuelle qui serait presque naturelle, essentielle, alors qu'en réalité ils en sont fort éloignés, si tant est qu'elle existe. C'est le cas notamment, dans les pays qui la reçoivent, avec l'immigration, puisque les immigrés sont souvent traités comme des " races dangereuses " ; c'est le cas également, qui éventuellement prolonge le précédent, avec la religion, à commencer par l'islam, couramment assimilé à l'islamisme. Ce dernier est souvent effectivement associé à des violences extrêmes, pouvant aller jusqu'au martyrisme mortifère des " bassidji " iraniens par exemple, si bien étudiés par Farhad Khosrokhavar ${ }^{9}$; mais il est aussi l'objet de soupçons qui témoignent éventuellement de l'aveuglement général d'une société sur elle-même. Ainsi, l'attentat d'Oklahoma City aux Etats-Unis (19 avril 1995, 168 morts, plusieurs dizaines de blessés) fut d'abord attribué massivement au terrorisme islamiste (qui avait récemment frappé à New 
York), avant que le pays, stupéfait, en découvre les auteurs, deux anciens militaires américains d'idéologie d'extrême-droite.

Par défaut: la violence, dans la mesure où elle s'inscrit dans le prolongement de problèmes sociaux classiques, ou qu'elle ne met pas en cause les modalités les plus fondamentales de la domination, est susceptible d'être niée ou banalisée. C'est ainsi, pour continuer avec l'exemple des Etats-Unis, que non seulement ce pays a peine à reconnaître sa violence interne contemporaine, mais a dû attendre les années soixante pour accepter de se pencher sérieusement sur certaines des pages les plus violentes de son histoire ${ }^{10}$; de même en France, les violences sociales des petits commerçants ou des agriculteurs, figures solidement installées à une place tout à fait respectable au coeur de l'imaginaire national, sont minimisées par rapport notamment à celles qui se jouent dans les quartiers de relégation, et qui sont elles l'objet d'une dramatisation et d'une amplification médiatique considérable, et éventuellement fantasmatique, sans base tangible. De même encore, il a fallu plusieurs années, toujours en France, pour que soit acceptée l'idée d'un lien entre le sentiment d'insécurité, puissant dans ce pays dès la fin des années soixante-dix, et la violence objectivement croissante de la criminalité, de la délinquance et surtout des petites incivilités : cette idée heurtait trop la sensibilité politique qui venait d'accéder aux affaires avec la première élection présidentielle de François Mitterrand (1981) pour pouvoir trouver un large espace, elle semblait trop liée aux représentations et au discours sécuritaire de la droite pour pouvoir être acceptée, et même entendue à gauche.

Non seulement la violence n'a guère aujourd'hui de légitimité dans l'espace public des démocraties occidentales, dans leurs débats politiques et intellectuels, dans leur capacité aussi à s'engager dans des interventions armées qui risqueraient de faire des morts de leur côté, mais de plus, il peut arriver qu'elle tienne lieu de mode de pensée général pour appréhender aussi bien la vie sociale que les relations internationales. Elle constitue alors une catégorie bien plus centrale qu'auparavant pour penser le dedans et le dehors, la société et son environnement. Le cas de la France est ici impressionnant, et peut-être même exceptionnel, puisque la violence y envahit les médias et l'opinion publique, qu'il s'agisse des banlieues et des quartiers de relégation, de l'école publique, des transports en commun, des incivilités qui nourrissent pour l'essentiel le sentiment d'insécurité, ou du terrorisme islamiste, dont les expressions les plus récentes permettent de fondre en un même sentiment l'image d'une menace intérieure, sociale, juvénile et urbaine, et celle d'une menace venue du dehors, religieuse et arabe ${ }^{11}$.

Il est possible qu'à l'avenir se rouvre l'espace d'une légitimité politique et intellectuelle de la violence, comme le suggère, encore très modestement, l'image de martyr qui caractérise désormais dans certains quartiers de relégation, Khaled Kelkal, un des protagonistes du terrorisme de l'été 1995 en France. De plus, comme nous le verrons plus loin, certaines violences sociales, celles d'agriculteurs en colère par exemple, sont à peine perçues comme telles, et bénéficient d'une large compréhension dans l'opinion publique. Par ailleurs, la remontée en puissance de courants de gauche protestataire, et la mise en forme intellectuelle qui va de pair, néo-gauchiste, sinon néo-marxiste, qui s'ébauchent dans certains pays, à commencer par la France, pourraient contribuer, là encore, à la réinvention de thématiques ouvertes à l'idée que la violence révolutionnaire, accoucheuse de l'histoire, apporte un mode de résolution des contradictions supposées être au coeur du système social. Enfin, et surtout, les perceptions et les représentations de la violence, évoquées ici surtout du point de vue 
de la France, changent considérablement d'une société à une autre, comme on le voit par exemple dans des pays où elle est tolérée ou supportée, perçue à la limite comme inscrite dans le fonctionnement normal de la société. Il en est ainsi, par exemple, du Brésil ou de la Russie. Mais dans l'ensemble, nous sommes bien éloignés des débats des années soixante et soixante-dix. approche totale de la violence, intégrant l'apport de toutes les disciplines des sciences sociales, ou humaines, psychologie, anthropologie, histoire, ce qui devait permettre d'aller de l'individu et de sa psychologie, jusqu'aux relations internationales - ce fut le projet de l'UNESCO, dont Pierre Hassner s'est fait récemment l'écho ${ }^{13}$, et qui devait donc assurer la compréhension, dans un même effort général, aussi bien des conflits entre père et fils, que des tensions naissantes de la guerre froide. Projet qui fut un échec. Et, si plusieurs raisonnements peuvent être repérés pour l'époque, les plus influents dans les années cinquante puis soixante oscillaient entre deux points de vue, éventuellement combinés. D'une part, la violence était liée à la notion de conflit; et d'autre part, elle était associée à l'image de la crise, et analysée alors comme la conséquence ou la manifestation d'un état plus ou moins pathologique du système considéré, par exemple du système social. Traiter de la violence, en effet, consistait ou bien à considérer qu'elle avait sa place dans les calculs et les stratégies des acteurs partie prenante d'un conflit, ou bien à admettre qu'elle venait traduire une insuffisante intégration des acteurs dans un système.

21

Dans le premier cas, la violence relevait d'interactions entre acteurs capables d'en user sur un mode instrumental; elle pouvait être pensée dans le contexte de modes $\mathrm{d}$ 'approche faisant appel à la théorie des jeux, ou à celle des ensembles organisés. Dans cette perspective, elle était un élément virtuel ou actuel dans le fonctionnement et les transformations de systèmes sociétaux ou intersociétaux, ce qu'illustrent bien les idées de Thomas Schelling ${ }^{14}$ qui exercèrent une influence considérable, donnant une grande importance à la rationalité des acteurs, et au fait que leurs décisions, y compris celle d'user de la violence, sont interdépendantes.

Dans le deuxième cas, la violence était largement pensée dans le cadre d'un néofonctionnalisme pour lequel elle venait traduire des dysfonctionnements, des carences, et leurs conséquences sur les acteurs, en termes de frustration relative par exemple ${ }^{15}$. Et pour certains chercheurs, ces carences et dysfonctionnements concernaient le conflit, la capacité des acteurs à fonctionner sur le mode du conflit, pensé comme 
nécessaire à l'intégration de la société, ou de tout système de relations internationales. L'idée était ici, comme le rappelle opportunément Pierre Hassner, que " les conflits peuvent, dans une certaine mesure, contribuer à l'intégration des systèmes ou organisations, et que l'intégration insuffisante de celles-ci peut être une source des conflits et de leur chute dans la violence "16.

Ce qui nous met en face de deux idées plus opposées que complémentaires. La première est que la violence peut s'inscrire, sur un mode avant tout instrumental, dans des relations, et relever de la communication et du rapport entre acteurs; la seconde est qu'elle peut venir traduire au contraire un déficit ou des difficultés dans les relations, la communication, le fonctionnement du rapport entre acteurs, ce qui fait qu'elle fonctionne alors surtout de manière expressive. Mais, il faut aussitôt ajouter deux remarques. La première est que la violence instrumentale peut être utilisée par un acteur pour tenter de pénétrer à l'intérieur d'un système de relations institutionnalisées - c'est là l'idée la plus centrale de la théorie dite de la mobilisation des ressources, à laquelle les noms de Charles Tilly et d'Anthony Oberschall sont particulièrement attachés ${ }^{17}$, et dont l'influence a été considérable dans les années soixante-dix et quatre-vingt. Et la deuxième remarque est que même à l'intérieur d'une relation structurelle ou systémique, le propre de la violence est d'avoir toujours une dimension qui déborde le cadre de la seule rationalité instrumentale, une dimension qu'on pourra appeler (un peu rapidement sans doute) irrationnelle, spontanée, ou expressive, et qui va au-delà du conflit - ce que percevait déjà Karl von Clausewitz lorsqu'il définissait la guerre comme " une étrange trinité composée de la violence originelle de son élément qu'il faut considérer comme une pulsion naturelle aveugle, du jeu de la probabilité et du hasard qui en font une libre activité de l'âme, et de la nature subordonnée d'un instrument politique, par quoi elle ressortit au pur entendement "18.

Pour prendre la mesure des principaux changements théoriques relatifs à l'analyse de la violence par les sciences sociales, le mieux est de considérer le chemin parcouru depuis l'époque où le phénomène pouvait être massivement et directement référé à des conflits, à leur fonctionnement ou à leur dysfonctionnements, ou à une crise. Aujourd'hui, l'analyse insiste de plus en plus sur deux types d'idées, sur lesquelles nous reviendrons, que résument des expressions comme individualisme, sujet, subjectivation d'un côté, éclatement, chaos, décomposition d'un autre côté. Ces idées suggèrent toutes un très grand éloignement par rapport aux deux notions, de conflit et de crise. La violence continue certes à être pensée dans des catégories où l'opposition entre instrumentalité et expressivité trouve sa place, mais ni son éventuelle instrumentalité, ni sa capacité à aller jusqu'aux pires extrémités, sans limites, ne renvoient à l'image d'un conflit, ou même à celle de la crise. Dans les cas extrêmes, elle semble s'autonomiser, devenir une fin en soi, ludique, purement destructrice ou autodestructrice par exemple. Ce qui en fait dans certains cas un phénomène de pure affirmation du sujet. L'analyse dès lors tend à dissocier le système et les acteurs, elle se centre plutôt sur l'un, ou plutôt sur les autres, et en tous cas elle constate leur séparation sans proposer l'image de médiations conflictuelles entre ces deux pôles de la réflexion, sans non plus donner à penser qu'elle traduit un simple dérèglement du système concerné - elle est bien davantage inscrite dans une mutation, un changement radical, ce qu'exprime d'ailleurs très bien le recours courant à des expressions en " post " (post-industriel, post-colonial, etc.), qui désignent toutes un dépassement, bien plus qu'un simple état d'un système. La violence, y compris dans ses expressions les plus 
localisées ou limitées, ou bien est expliquée par des changements planétaires, la globalisation de l'économie, ou la fin de la guerre froide, thèmes sur lesquels nous reviendrons; ou bien elle est réduite aux calculs ou, ce qui n'est pas du tout du même ordre, à la subjectivité des acteurs, éventuellement à leur folie. Et entre les deux registres, celui du système et celui de l'acteur, la pensée insiste sur ce qui est défait, liquidé, les rapports sociaux de l'ère industrielle, le système bipolaire des relations internationales jusqu'à la chute du mur de Berlin, par exemple, bien plus qu'elle n'est capable de dessiner l'image de conflits, ou même de processus de déstructuration de rapports conflictuels, ou bien encore celle de dysfonctionnements systémiques.

Pour qu'il y ait conflit, système d'acteurs en relations conflictuelles, il faut d'une part des acteurs, d'autre part des enjeux qu'ils reconnaissent comme communs, et enfin des possibilités pour eux de s'opposer sans se détruire, et donc des mécanismes politiques ou institutionnels. Et pour qu'on puisse parler de crise, il faut qu'il y ait un système, en difficulté certes, mais qui demeure encore perceptible comme tel. Si la violence semble aujourd'hui si menaçante ou dramatique, n'est-ce pas du fait de la démultiplication des " anti-acteurs ", protagonistes, extérieurs à tout système d'action, d'une violence relevant de la seule logique de la force et de la puissance, sans débat ni relation possible avec eux? N'est-ce pas aussi en raison des carences relatives aux procédures et processus qui permettent le fonctionnement du conflit, la relation; n'est-ce pas encore parce que les systèmes qui ont fonctionné depuis la fin de la Deuxième Guerre mondiale, sociaux, ou internationaux, font plus que se transformer, ils se défont, au point que la notion de crise est trop faible pour rendre compte de leur déstructuration? Mais n'est-ce pas aussi parce que des personnes et des groupes se vivent comme niés, interdits de subjectivité, cassés ou détruits par le mépris d'autres personnes et groupes mieux placés qui refusent de les reconnaître comme sujets?

Bien que d'importantes distinctions opposent les pensées les mieux établies aujourd'hui, elles présentent un point commun : la plupart considèrent que le monde est de plus en plus un univers sans acteurs, et tenté par la seule loi de la jungle que constitue le marché, par le chaos ou par le choc des identités et des cultures, bien plus que par des rapports plus ou moins négociés impliquant un minimum de reconnaissance mutuelle. Qu'il s'agisse, à l'intérieur des sociétés, du fonctionnement du système des partis, avec un clivage gauche-droite qui souvent s'est brouillé, de l'affaiblissement assez général du syndicalisme et des systèmes de relations professionnelles, des modes de gestion de l'Etat-providence et, plus largement, de l'ensemble des modes de relations qu'ont inventés les sociétés industrielles depuis le XIXe siècle; qu'il s'agisse, dans les relations internationales, du fonctionnement bipolaire que structurait l'opposition entre les Etats-Unis et l'Union soviétique, ou des échecs réitérés des grandes organisations internationales, à commencer par l'ONU et ses Casques bleus en Bosnie, en Somalie ou au Liban, comment ne pas voir qu'effectivement, les procédures et mécanismes institutionnels laissent souvent la place au seul usage de la force? Tout n'est pas ou plus conflictualisable dans ce qui pouvait l'être auparavant. Ainsi, certains chercheurs parlent de " clash " des civilisations, emboîtant le pas à Samuel Huntington ${ }^{19}$, d'autres, de façon plus pertinente, constatent que d'intenses tensions interculturelles opèrent au sein même des " civilisations ", et pas seulement entre elles ${ }^{20}$; certains voient le monde comme unipolaire, avec les Etats-Unis comme unique puissance, d'autres le perçoivent comme totalement éclaté, voire sur la voie du chaos généralisé et moléculaire, d'autres encore cherchent à dessiner une multipolarité, ce qui correspond à d'autres représentations 
des Etats-Unis. Mais au-delà de ces débats ${ }^{21}$, qui parle encore d'acteurs collectifs capables de s'engager dans des conflits où la négociation et les jeux politiques à la Schelling trouveraient un espace important? Qui résiste aux images, assurément fortes et prégnantes, de la violence comme expression, précisément, de l'incapacité de l'ère actuelle à mettre en place et à faire fonctionner des systèmes d'acteurs? Difficile à remplir, par la pensée, d'acteurs et de conflits, le monde est peuplé par les images et les craintes de la violence et de l'insécurité, avec toutes les dérives que peuvent encourager ces images et ces craintes, et notamment la démonisation de l'Autre qui voit par exemple un terroriste derrière chaque musulman - d'ailleurs désormais, en France, on utilise de moins en moins le qualificatif de " musulman " et de plus en plus couramment celui d' "islamiste ".

Ainsi, aussi bien comme réalité historique, que comme représentation collective et comme objet d'analyse et de réflexion pour les sciences sociales, la violence contemporaine semble bien modeler un nouveau paradigme. Du point de vue théorique, ce paradigme appelle que la violence soit analysée dans un espace théorique complexe, qui intègre mais déborde aussi bien le champ du conflit que celui de la crise, pour s'élargir, d'un côté vers la prise en compte du sujet, impossible, frustré ou fonctionnant hors système et hors normes, et d'un autre côté vers celle de conduites qui, au-delà de la crise, témoignent d'une véritable déstructuration ou de dérives menant au chaos et à la barbarie.

\section{NOTES}

1. Cf. par exemple Charles Tilly, La France conteste de 1600 à nos jours, Paris, Fayard, 1986.

2. Sur ce point, cf. par exemple Yves Michaud, La violence apprivoisée, Paris, Hachette, 1996.

3. Sur les " Apaches ", ces jeunes dont les bagarres et les conduites délinquantes font penser à certains égards aux jeunes des quartiers actuels de relégation lorsqu'ils ont la rage ou la haine, et dont l'expérience a été immortalisée au cinéma par le personnage de " Casque d'or " interprété par Simone Signoret, cf. Régis Pierret, Les Apaches, Paris, Diplôme de l'EHESS, 1996.

4. Cf. Michel Wieviorka, " Quatre figures du nationalisme : la question de la violence ", dans Pierre Birnbaum (ss la dir. de), Sociologie des nationalismes, Paris, PUF, 1997, p. 369-386.

5. | Jean-François Bayart, L'illusion identitaire, Paris, Fayard, 1996, p. 10.

6. Jean Baudrillard, " Le degré Xerox de la violence ", dans Libération, 2 octobre 1995.

7. Frantz Fanon, Les damnés de la terre, Paris, Maspéro, 1961, préface de Jean-Paul Sartre.

8. Cf. Philippe Gavi, Jean-Paul Sartre, Pierre Victor, On a raison de se révolter, Paris, Gallimard, 1974. 
9. Farhad Khosrokhavar, Rupture de l'unanimisme dans la révolution iranienne, thèse pour le doctorat d'Etat, Paris, EHESS, 1992. Voir aussi l'étude qu'il propose dans ce numéro de Cultures et Conflits.

10. Cf. Yves Michaud, Violence et politique, Paris, Gallimard, 1978, qui rappelle toute l'importance de la Commission mise en place en 1968 à la demande du Président Lyndon Johnson avec pour tâche d'aller aussi loin que la connaissance peut le faire dans la recherche des causes de la violence et des moyens de la prévenir, et pour projet de " découvrir la violence dissimulée sous l'histoire paisible des Etats-Unis ".

11. Sur ce terrorisme et ses effets sur le fonctionnement de la démocratie et l'Etat de droit, on me permettra de renvoyer à mon livre Face au terrorisme, Paris, Liana Lévi, 1995.

12. Cf. notamment James B. Rule, Theories of Civil Violence, Berkeley, University of California Press, 198 ; et, en français, l'annexe théorique de mon livre Sociétés et terrorisme, Paris, Fayard, 1988.

13. Pierre Hassner, La violence et la paix. De la bombe atomique au nettoyage ethnique, Paris, Ed. Esprit, 1995, cf. p. 83-84, qui reprennent un texte de 1964.

14. Cf. notamment Thomas Schelling, The Strategy of Conflict, New York, Galaxy Book, 1963.

15. Cf. ce qui fut la tentative la plus ambitieuse, et qui est venue en fait clore cette époque, Ted Robert Gurr, Why Men Rebel ?, Princeton, Princeton University Press, 1970.

16. Pierre Hassner, op. cit., p. 90.

17. Cf. notamment Charles Tilly, From Mobilization to Revolution, Addison-Wesley, Reading, Mass., 1978 ; Anthony Oberschall, Social Conflict and Social Movements, Prentice Hall, Englewood Cliffs, New Jersey, 1972.

18. Dans De la Guerre, I, 28, cité par Pierre Hassner, op. cit., p. 37.

19. Samuel Huntington, " The Clash of Civilizations ", in Foreign Affairs, summer 1993

20. Cf. Yvon Le Bot, " Le temps des guerres communautaires ", dans Michel Wieviorka (dir.), Une société fragmentée ? Le multiculturalisme en débat, Paris, La Découverte, 1996, p. 173-197.

21. Pour une utile mise en perspective à partir de l'expérience américaine, cf. John Mason, " Le désalignement du consensus stratégique américain ", Le débat stratégique américain 1994-95, in Cahiers d'Etudes Stratégiques, 18, 1995, p. 21-55.

INDEX

Mots-clés : sociologie, sociologie des conflits (polémologie), violence 